\title{
A child, homozygous for a stop codon in exon 11, shows milder cystic fibrosis symptoms than her heterozygous nephew
}

\author{
H Cuppens, P Marynen, C De Boeck, F De Baets, E Eggermont, H Van den Berghe, J J Cassiman
}

\begin{abstract}
The clinical and molecular findings in an infant with mild manifestations of cystic fibrosis, who is homozygous for the G542X mutation, and her heterozygous nephew, who is severely affected, are described.
\end{abstract}

The major mutation recently identified in the CFTR gene $^{1-3}$ is a specific deletion of three base pairs, which results in the loss of a phenylalanine residue at amino acid position $508(\Delta F 508){ }^{3}$ In a sample of Belgian CF patients, $68 \cdot 1 \%$ of all CF chromosomes (94 out of 138 CF chromosomes) carried this mutation. ${ }^{4}$

Since then, a series of rarer mutations has been reported (CF genetic analysis consortium, personal communication), including the 2566ins $\mathrm{AT}^{5}$ and the G542X mutation (Kerem et al, personal communication). The 2566insAT mutation was not present in our Belgian population sample. In our CF patients, the G542X mutation, in which the glycine (GGA) at amino acid position 542 in exon 11 is mutated to a stop codon (TGA), accounted for $7 \cdot 3 \%$ of the CF chromosomes, being probably the second most frequent $\mathrm{CF}$ mutation in this population sample.

Here we describe the clinical and molecular findings in a child who is only mildly affected although homozygous for the mutation, and a child who is

Centre for Human Genetics, University of Leuven, Campus Gasthuisberg 0 \& $\mathbf{N}$, Herestraat 49, B-3000 Leuven, Belgium.

H Cuppens, $\mathbf{P}$ Marynen, H Van den Berghe, J J Cassiman

Department of Pediatrics, UZ Gasthuisberg, Leuven, Belgium.

C De Boeck, E Eggermont

Kliniek.voor Kinderziekten 'C Hooft', University Hospital, Gent, Belgium.

F De Baets

Correspondence to Professor Cassiman.

Received for publication 22 May 1990.

Accepted for publication 18 June 1990. severely affected and heterozygous for the G542X mutation and a new mutation in exon 9 (G458V).

\section{Case report}

II.1 (fig 1) is the oldest of three sibs born to healthy, unrelated, Belgian parents. The diagnosis of cystic fibrosis was made by a positive sweat chloride test at the age of 3 years, after the diagnosis in his younger niece (II.2). Initially the gastrointestinal problems, especially malabsorption, were more pronounced than the respiratory symptoms.

By the age of 9 years the respiratory insufficiency was pronounced $\left(\mathrm{pH} 7 \cdot 44, \mathrm{PCO}_{2} 52 \mathrm{mmHg}, \mathrm{Po}_{2}\right.$ $48 \mathrm{mmHg}, \mathrm{HCO}_{3} 35$, basic excess +10 ) and he showed signs of cor pulmonale on ECG and electrocardiography. Sputum cultures grew Pseudomonas.

Pulmonary function test showed a total lung capacity $112.5 \%$ of normal, vital capacity $24 \%$, functional residual capacity $194 \%$, and residual volume $358 \%$ of normal. The child died at the age of 12 years of respiratory insufficiency and right heart failure.

II. 2 is the oldest of three sibs born to healthy, unrelated, Belgian parents. She was diagnosed as having cystic fibrosis at the age of 2 years because of a history of failure to gain weight in the presence of

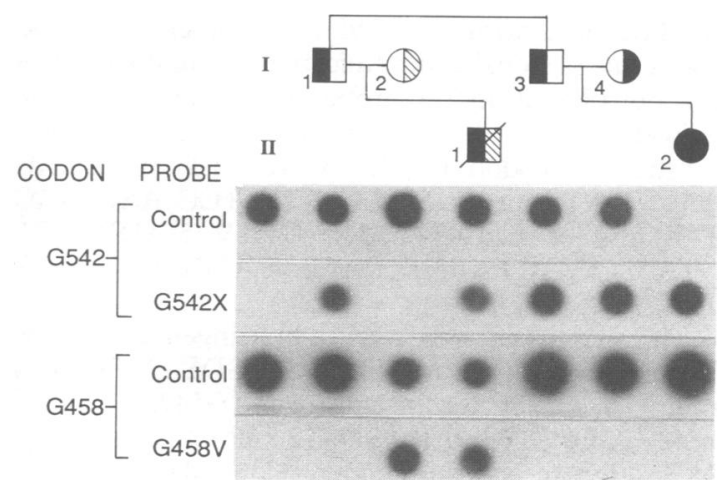

Figure 1 Detection of the G542X and G458V mutations by allele specific oligonucleotides. The first sample in each row is a normal control. 
copious fatty stools, rectal prolapse, and a positive pilocarpine sweat chloride test. Once treatment with pancreatic enzyme supplements, vitamin supplements, and daily chest physiotherapy was instituted, she thrived well. Intermittent cough and daily sputum production owing to respiratory tract disease started at the age of 5 years. Staphylococcus aureus or Haemophilus influenzae or both were found in the sputum throughout childhood. Pseudomonas aeruginosa was recovered from the sputum from the age of 10 years onwards. She was admitted to hospital at the age of $11 \frac{1}{2}$ years because of fever, cough, increased sputum production, and weight loss of several weeks duration. Chest $x$ ray showed hyperinflation as well as inhomogeneous infiltrates in the lingula and right middle lobe. Sputum culture grew $2+$ Pseudomonas aeruginosa resistant to tobramycin and piperacillin. Ceftazidim was administered intravenously for 14 days and the girl recovered well. The pulmonary infiltrates resolved completely. At present she is $121 / 2$ years old and she continues to do very well. She is very active in sport. She produces a moderate amount of yellowish sputum a day (about 10). She has two or three bowel movements a day and no abdominal complaints. Her height is $160 \mathrm{~cm}$ and weight is $51.4 \mathrm{~kg}$, both between the 75th and 97th centile. Physical examination is normal and there is no obvious hyperinflation, normal lung auscultation, and no abdominal distension or hepatosplenomegaly. Tanner pubertal score is M2P2A2. Sputum culture consistently grows Pseudomonas aeruginosa. Pulmonary function tests are excellent: vital capacity $117 \%$ of normal, forced expiratory volume in one second $120 \%$ of normal, Tiffneau index 85 , thoracic gas volume $117 \%$, as well as normal flow rates at low lung volumes and normal airway resistance. Chest $x$ ray shows mild hyperinflation and mild increase in bronchovascular markings. Shwachman score is 90/100.

Genomic DNA extracted from peripheral blood of all members of the family was amplified by PCR with the $11 \mathrm{i} 5,11 \mathrm{i} 3$ primers and $9 \mathrm{i} 5,9 \mathrm{i} 3$ primers (Zielenski and Tsui, personal communication). Amplified DNA was dot blotted on Zetaprobe. The membranes were hybridised with ${ }^{32} \mathrm{P}$ labelled oligonucleotide probes: for the G542 codon (G542X: 5'-ACC TTC TCA AAG AAC T-3'; control: 5'ACC TTC TCC AAG AAC T-3') and for the G458 codon (G458V: 5'-GTT GCT GTA TCC ACT G-3'; control: 5'-GTT GCT GGA TCC ACT G-3').

The membranes were washed in stringent conditions $\left(10\right.$ mins at $42^{\circ} \mathrm{C}$ in $6 \times \mathrm{SSC}, 0.1 \%$ SDS; $2 \times 30$ mins at $45^{\circ} \mathrm{C}$ in $2 \times S S C$ for the control and G542X, 10 mins at $42^{\circ} \mathrm{C}$ in $6 \times \mathrm{SSC}, 0.1 \% \mathrm{SDS} ; 2 \times 30 \mathrm{mins}$ at $56^{\circ} \mathrm{C}$ in $2 \times \mathrm{SSC}$ for the control of codon $\mathrm{G} 458,10 \mathrm{mins}$ at $42^{\circ} \mathrm{C}$ in $6 \times \mathrm{SSC}, 0.1 \% \mathrm{SDS} ; 2 \times 30 \mathrm{mins}$ at $52^{\circ} \mathrm{C}$ in $2 \times \mathrm{SSC}$ for $\mathrm{G} 458 \mathrm{~V}$ ) and autoradiographed for three hours at $-70^{\circ} \mathrm{C}$.
Sequencing of the fragment containing exon 9 was done with the T7 sequencing ${ }^{\mathrm{TM}}$ kit (Pharmacia) according to the following modified protocol. The amplified fragments were purified with GeneClean (Biol01) and were mixed with $15 \mathrm{ng}$ of an internal end labelled primer (5'-CAT GGA CAC CAA ATT AAG TTC- $\left.3^{\prime}\right)$. The fragments were denatured for three minutes at $100^{\circ} \mathrm{C}$ and cooled on ice. Annealing buffer, labelling mix, dATP, and T7 DNA polymerase were added together, mixed, and the total reaction mixture was immediately split into four tubes containing the dideoxymixes (short). The sequencing reactions were kept for five minutes at room temperature, then for

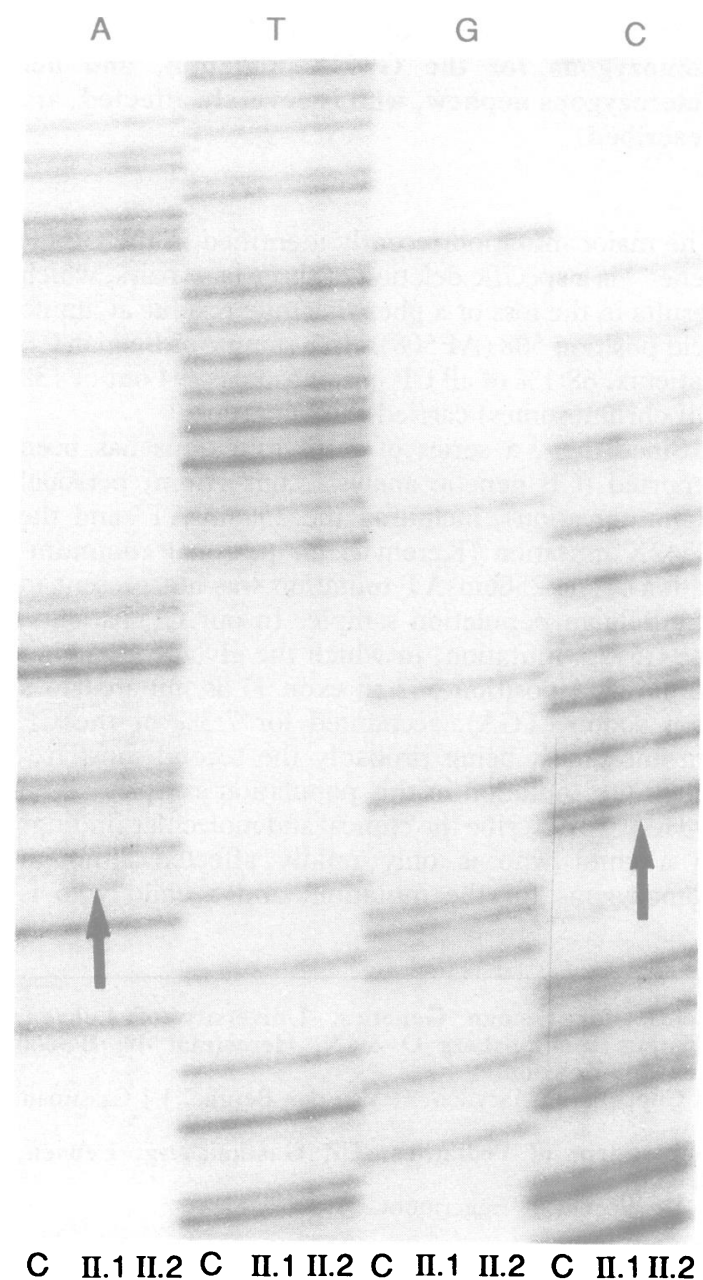

Figure 2 Detection of the mutation $G 458 \mathrm{~V}$ by direct sequencing of exon 9. To allow for direct comparison, the termination reactions for each nucleotide (respectively $d d A, d d T, d d G$, and $d d C$ ) of the different samples were loaded in adjacent lanes. Lane I control, lane 2 II.1, and lane 3 II.2. The mutation $(C \rightarrow A)$ is indicated by an arrow. 
five minutes at $37^{\circ} \mathrm{C}$ and stop solution was added. The sequencing reaction products were then separated on a $6 \%$ acrylamide $/ 8 \mathrm{~mol} / \mathrm{l}$ urea gel. The gel was fixed in $10 \%$ acetic acid $/ 10 \%$ methanol solution, dried, and exposed.

Dot blot analysis (fig 1) showed that the CF girl (II.2) was homozygous and her cousin (II.1) heterozygous for the G542X mutation. Direct sequencing of an amplified fragment, containing exon 9, of patient II.1 detected a new mutation (fig 2), resulting in a substitution of a conserved glycine (GGA) for a valine (GTA) at amino acid position 458 (G458V). This was confirmed by dot blot analysis (fig 1). This mutation segregates with the haplotype 21111 (XV2c, CS7, $\mathrm{KM} 19$, D9). This amino acid substitution is most likely a disease mutation rather than a polymorphism because glycine is a very conserved amino acid in the nucleotide binding fold of this region and this substitution was not detected in 45 normal chromosomes, 20 of them with the same haplotype 21111 (XV2c, CS7, KM19, D9). This mutation has been found in one out of $31 \mathrm{CF}$ chromosomes, which carry a so far unknown mutation. Three other CF chromosomes with this haplotype did not carry this mutation and therefore at least one other mutation might be associated with this haplotype. This mutation has not been found in $21 \Delta \mathrm{F} 508$ chromsomes, one $\Delta \mathrm{I} 507$ chromosome (CF genetic analysis consortium), or in two G542X chromosomes.

The G542X mutation segregates with the haplotype 1222 (XV2c, CS7, KM19, D9) and was found in 10 out of 41 non- $\Delta \mathrm{F} 508$, non- $\Delta \mathrm{I} 507 \mathrm{CF}$ chromosomes. Since $68 \cdot 1 \%$ of all CF chromosomes carry the $\Delta F 508$ deletion in our population sample, the $\mathrm{G} 542 \mathrm{X}$ mutation accounts for $7 \cdot 3 \%$ ( 10 out of $138 \mathrm{CF}$ chromosomes) and the G458V mutation accounts for less than $1 \%$ (one out of $138 \mathrm{CF}$ chromosomes) of all the CF chromosomes in this sample.

Based on the quite mild clinical picture, one would have expected that the CF girl carried a mutation in a non-essential part of the gene. The G542X mutation, however, creates a stop codon at amino acid 542 . Therefore, only the first 541 codons, out of 1480 codons, would be translated, resulting in a CFTR protein composed of the first membrane spanning segment and half of the first putative nucleotide binding fold (including only two out of the three very conserved regions ${ }^{2}$ ). Splicing events, which would jump this mutation and allow the synthesis of an almost normal protein, cannot be ruled out, however.

In contrast, the nephew who was heterozygous for the G458V mutation and shares the G542X mutation with II.2, suffered from an apparently severe form of CF. This would suggest the G458V mutation might be a rather severe mutation.

Experiments directed towards examination of the CFTR mRNA of the homozygous G542X patient (II.2) and protein analysis using antibodies to different domains of the CF protein will allow the study of the exact structure of the synthesised proteins and their insertion as 'truncated' molecule, as dimer, or as alternatively spliced protein in the cell membrane, while patch clamp studies should allow one to evaluate the functional properties of the protein. This may be helpful in the analysis of structure-function relations of the CFTR protein and provide some insight into the complexity of the pathophysiology of CF.

The authors are grateful for the generous collaboration of the family members described here. The technical assistance of Mariëlle Braeken and Katrien Van den Bosch is gratefully acknowledged. This work was supported by the Inter-University Network for Fundamental Research sponsored by the Belgian Government (1987-1991).

1 Rommens JM, Iannuzzi MC, Kerem B-S, et al. Identification of the cystic fibrosis gene: chromosome walking and jumping. Science 1989;245: 1059-65.

2 Riordan JR, Rommens JM, Kerem B-S, et al. Identification of the cystic fibrosis gene: cloning and characterization of complementary DNA. Science 1989;245:1066-73.

3 Kerem B-S, Rommens JM, Buchanan JA, et al. Identification of the cystic fibrosis gene: genetic analysis. Science 1989;245: $1073-80$.

4 Cuppens H, Legius E, Cabello P, et al. Association between XV2c/CS7/LM19/D9 haplotypes and the $\Delta$ F508 mutation. A study of 57 Belgian families. Hum Genet (in press).

5 White MB, Amos J, Hsu JMC, et al. A frame-shift mutation in the cystic fibrosis gene. Nature 1990;344:665-7. 